Original Research Paper

\title{
Exploring Knowledge and Attitude toward Middle East Respiratory Syndrome-Coronavirus (MERS-CoV) Among University Health Colleges' Students, Saudi Arabia: A Cross- Sectional Study
}

\author{
${ }^{1}$ Ahmed Morad Asaad, ${ }^{1}$ Rehab H. El-Sokkary, ${ }^{2}$ Abdullah I. Aedh, \\ ${ }^{2}$ Mahdi Aly Ali Alzamanan and ${ }^{3}$ Fatma Omar Khalil \\ ${ }^{1}$ Department of Medical Microbiology and Immunology, Faculty of Medicine, Zagazig University, Zagazig, Egypt \\ ${ }^{2}$ Department of Medicine, College of Medicine, Najran University, Najran, Saudi Arabia \\ ${ }^{3}$ Department of Medical Microbiology and Immunology, National Liver Institute, Menofia University, Menofia, Egypt
}

Article history

Received: 11-11-2018

Revised: 27-02-2019

Accepted: 09-03-2019

Corresponding Author: Ahmed Morad Asaad

Department of Medical Microbiology and Immunology,

Faculty of Medicine, Zagazig

University, Zagazig, Egypt

Email: ahmedmoradassad@hotmail.com

\begin{abstract}
Middle East Respiratory Syndrome Coronavirus (MERS-CoV) is an emerging pathogen with a pandemic potential and a higher burden in Saudi Arabia. This cross-sectional study aimed to investigate the knowledge and attitude toward MERS-CoV among Health Colleges' students in a Saudi public university. The study included 540 students. A well-constructed questionnaire containing 14 knowledge and 8 attitude items was completed by all participants. Overall, $>80 \%$ of students were aware about MERS-CoV aetiology, mode of transmission, risk factors and signs and symptoms. Knowledge scores revealed that $42 \%$ of participants have sufficient knowledge. Medical students had significantly better knowledge compared to students in other Health Colleges ( $p$ $=0.001$ ). Concerning attitude, $>50 \%$ of students exhibited a positive attitude toward MERS-CoV. This study showed a good knowledge level and a strong positive attitude towards protection from MERS-CoV among Health Colleges' students. However, there is a noticeable defect in the knowledge level about the microbiology of the virus and the infection control measures. The findings of this study highlight the need to design educational courses on new emerging diseases, field epidemiology, infection control practice and public health to all Health Colleges' students to prepare them for dealing with these types of health emergencies in their future healthcare practice. Besides, continuous awareness campaigns about new emerging diseases are warranted for all Health Colleges' students to improve their knowledge and attitude toward emerging infection trends.
\end{abstract}

Keywords: MERS-CoV, Knowledge, Attitude, Students, Saudi Arabia

\section{Introduction}

Middle East Respiratory Syndrome Coronavirus (MERS-CoV) is an emerging pathogen with a pandemic potential and a noticeable epicenter in the Kingdom of Saudi Arabia (Khan et al., 2013). In September 2012, the first case (the index case) reported in Saudi Arabia was presented by pneumonia and acute kidney injury symptoms (Zaki et al., 2012). Soon after that, the disease appeared in other Arab peninsula countries such as Qatar, United Arab Emirates, Kuwait and then spread to Middle East and non-Middle East countries (European Centre for Disease Prevention and Control, 2015). However all cases reported from outside the Middle East have a history of recent travel to the Middle East or linked to a case of traveling history (CDC, 2015).

So far, no vaccine has been developed for the MERS$\mathrm{CoV}$ and no antiviral treatment is specifically recommended. Therefore, applying preventive measures to reduce the spread of the disease is of utmost importance. For any preventive recommendations to be beneficial, a comprehensive approach must be taken to ensure the proper health education of the public (AlHazmi et al., 2018). Great efforts were exerted by the health authorities. The Saudi Ministry of Health (MOH) website provides educational health tips regarding the frequent inquiries, prevention of transmission and dealing with infected cases (Saudi Ministry of Health, 
2018). Moreover, during Hajj season, the MOH provides courses and holds comprehensive symposiums for medical students concerning transmissible diseases, as the role of medical students is undoubtedly substantial in spreading the awareness among their families, friends and communities (Khairoalsindi et al., 2017).

Increased MERS-CoV's prevalence and the occurrence of asymptomatic and subclinical MERS-CoV in healthcare settings represent a huge threat to public health. In turn, health care workers (HCWs) and medical students are at great risk of acquiring and subsequently transmitting this lethal virus to their patients, families and colleagues (Memish et al., 2014).

Health authorities must ensure that all Health Colleges' students are capable of practicing safely concerning infection control measures before starting their practical sessions at the hospitals and health centers (Ibrahim and Alghamdi, 2016). Recent epidemiological research has highlighted a number of psychiatric morbidities associated with exposure to devastating epidemics of novel and highly lethal viruses. For medical professionals, the psychological effects of such exposure can jeopardize the medical care they provide (Aldrees et al., 2017). Thus understanding the attitude of medical students that will be in close contact with MERS patients may help in preparing them to face any upcoming MERS-CoV cases or outbreaks.

Many previous studies were conducted to investigate the knowledge and attitude of students in health colleges at different universities (Al-Hazmi et al., 2018; Al-Mohrej and Agha, 2017; Hassan, 2016; Khairoalsindi et al., 2017). However, more studies, in different geographical locations, are still needed. Evidence based data from such studies could help health authorities and policy decision makers in higher education systems to design appropriate strategies in improving the awareness and attitude towards the MERS-CoV. The aim of this study was to investigate knowledge and attitude towards MERS-CoV among Health Colleges' students in a Saudi University.

\section{Materials and Methods}

This cross-sectional descriptive study of a representative sample of Health Colleges' students in Najran University, Saudi Arabia was conducted from January to June 2017. The study was conducted according to the international guidelines of Strengthening the Reporting for Observational Studies in Epidemiology; STROBE (STROBE checklist, 2007). Using the WHO Manual for Sample Size Determination in Health Studies (Lwanga and Lemeshow, 1990), the minimal sample size calculated for the study was 490 students based on an average previous estimate of 54\% MERS knowledge among Saudi public (Al-Mohrej et al., 2016) and with an absolute precision of $2 \%$ and at a $95 \%$ confidence interval. To avoid loss of participants, a total sample of 540 students was included in the present study. The study followed the principles of the Helsinki Declaration and ethics approval was obtained from the Ethics and Research committee of Najran University. Written consent was obtained from all participants.

\section{Questionnaire Interview}

A well-constructed standardized self-administered questionnaire was distributed as hard copies for all participants. Interviews were performed in each health college with all students to explain the purpose of the study and to give instructions for the questionnaire to be filled anonymously. The questionnaire was developed with some modifications using the frequently asked questions from the Saudi MOH and the World Health Organization (WHO) websites (Saudi Ministry of Health, 2018; WHO, 2018).

The questionnaire was initially designed in English and translated into Arabic by experts of infectious diseases and biostatistics to fit with local colloquial Arabic terminology of Saudi Arabia used by physicians and health educators in the community. After translation and back translation, a panel of experts was asked to assess the preliminary questions and provide structured comments with respect to face and content validity, comprehensibility and comprehensiveness. The questionnaire was composed of three parts to address knowledge and attitude of health colleges' students regarding MERS-CoV. The first part included information about the demographic data such as age, sex, type of Health College, type of house and participant's source of MERS knowledge. The second part assessed the knowledge of students by asking questions about the etiology, incubation period, symptoms, risk group, consequences, source of transmission, prevention and treatment of MERS-CoV. A scoring system was applied to assess the level of knowledge of each subject (Two points were given for each correct answer and one point was given for an incorrect answer). Participants were grouped into two categories according to their level of knowledge: Insufficient ( $<12$ points) and sufficient $(>12$ points). The third part assessed the attitude of students regarding MERS in which Yes or No option was given against a set of 8 questions.

\section{Statistical Analysis}

Data were coded, validated and analyzed using the Statistical Package for the Social Sciences (SPSS), version 22 (SPSS Inc., Chicago, IL, USA). Frequencies and proportions were used to present the data. Chi square and the $t$ test were used as tests of significance at 5\% level. 


\section{Results}

The study included 540 students with an age range of 23-29 years and a median of 23 years. More than three quarters of the respondents $(77 \%)$ were male. Table 1 shows the baseline characteristics of the respondents. More than half of the respondents $(58 \%)$ perceive that they have sufficient knowledge about MERS. The main sources of MERS information were social media (57\%) and $\mathrm{MOH}$ website (32\%).

Table 2 shows the current status of MERS knowledge among students. The majority $(90 \%)$ were aware that the disease is a viral infection, with $82 \%$ and $91 \%$ being familiar with its mode of transmission and signs and symptoms, respectively. Nevertheless, less than half (48\%) of students reported that diarrhea is a possible symptom of MERS. Of all participants, $61 \%$ and $67 \%$ agreed that patients with chronic diseases and HCWs are at a higher risk of infection, respectively. The participants' knowledge about other questions related to the MERS-CoV vaccination and treatment by antibiotics was poor (54\%).

In this study, the knowledge scores were studied in relation to the baseline characteristics of the respondents (Table 3). Of all participants, $42 \%$ had sufficient knowledge. Overall, sufficient knowledge was significantly correlated with age $(p=0.001)$. Moreover, students in the college of medicine had significantly better knowledge compared to students in other colleges $(p=0.001)$.

Table 1: The baseline characteristics of Health Colleges' students

\begin{tabular}{ll}
\hline Variable & Participants (n. $=540)$ N. (\%) \\
\hline Sex: & $416(76.7)$ \\
Male & $124(23.3)$ \\
Female & \\
Age (Years): & $95(17.6)$ \\
$\leq 20$ & $400(74.1)$ \\
$21-25$ & $45(8.3)$ \\
$26-30$ & \\
Health college: & $118(21.9)$ \\
Medicine & $86(15.9)$ \\
Dentistry & $80(14.8)$ \\
Pharmacy & $82(15.1)$ \\
Physiotherapy & $104(19.3)$ \\
Radiology & $70(13)$ \\
Medical laboratory & \\
Type of house: & $196(36.3)$ \\
Flat & $142(26.3)$ \\
Popular house & $175(32.4)$ \\
Villa & $27(5)$ \\
Others & \\
Do you have sufficient knowledge about MERS-CoV? & $315(58.3)$ \\
Yes & $220(40.7)$ \\
No & $5(1)$ \\
Don't know & \\
Source of knowledge about MERS-CoV: & $175(32.4)$ \\
MOH website & $308(57)$ \\
Social media & $40(7.4)$ \\
TV & $17(3.2)$ \\
Newspaper & \\
\hline
\end{tabular}

Table 2: Knowledge of Health Colleges' students about MERS-CoV

\begin{tabular}{ll}
\hline Question & Correct answer N. (\%) \\
\hline MERS-CoV is a viral infection & $488(90.4)$ \\
MERS-CoV is transmitted by close contact with infected person or animal & $442(81.9)$ \\
Fever, cough and shortness of breath are symptoms of MERS & $490(90.7)$ \\
Diarrhea is a possible symptom of MERS & $260(48.1)$ \\
The incubation period is 2-4 weeks & $276(51.1)$ \\
MERS-CoV vaccine is available in markets & $290(53.7)$ \\
Antibiotics are the first-line treatment & $293(54.3)$ \\
Washing hands with soap and water can help in prevention of disease transmission & $415(76.9)$ \\
Patients with underlying chronic diseases at a higher risk of infection & $331(61.3)$ \\
Healthcare workers are at a higher risk of infection & $360(66.7)$ \\
MERS could be fatal & $338(62.6)$
\end{tabular}


Table 3: Knowledge score among Health Colleges' students

\begin{tabular}{|c|c|c|c|}
\hline Variable & $\begin{array}{l}\text { Sufficient knowledge } \\
(\mathrm{n} .=255) \text { N. }(\%)\end{array}$ & $\begin{array}{l}\text { Insufficient knowledge } \\
(\mathrm{n} .=285) \mathrm{N} .(\%)\end{array}$ & $\mathrm{p}$-value \\
\hline \multicolumn{4}{|l|}{ Sex: } \\
\hline Male & $192(46.2)$ & $224(53.8)$ & \multirow[t]{2}{*}{0.982} \\
\hline Female & $63(52.5)$ & $61(47.5)$ & \\
\hline \multicolumn{4}{|l|}{ Age (Years): } \\
\hline$\leq 20$ & $35(36.8)$ & $60(63.2)$ & \multirow[t]{3}{*}{0.001} \\
\hline $21-25$ & $199(49.8)$ & $201(50.2)$ & \\
\hline $26-30$ & $21(70)$ & $9(30)$ & \\
\hline \multicolumn{4}{|l|}{ Health college: } \\
\hline Medicine & $86(72.9)$ & $32(27.1)$ & \multirow[t]{6}{*}{0.001} \\
\hline Dentistry & $30(34.9)$ & $56(65.1)$ & \\
\hline Pharmacy & $27(33.8)$ & $53(66.2)$ & \\
\hline Physiotherapy & $28(34.1)$ & $54(65.9)$ & \\
\hline Radiology & $48(46.2)$ & $56(53.8)$ & \\
\hline Medical laboratory & $36(51.4)$ & $34(48.6)$ & \\
\hline \multicolumn{4}{|l|}{ Type of house: } \\
\hline Flat & $78(39.8)$ & $118(60.2)$ & \multirow[t]{4}{*}{0.216} \\
\hline Popular house & $75(56.8)$ & $67(43.2)$ & \\
\hline Villa & $90(38.4)$ & 85 (61.6) & \\
\hline Others & $12(44.4)$ & $15(55.6)$ & \\
\hline \multicolumn{4}{|l|}{ Presence of animals } \\
\hline Yes & $94(57.3)$ & $70(42.7)$ & \multirow[t]{3}{*}{0.117} \\
\hline No & $152(46.5)$ & $175(53.5)$ & \\
\hline Don’t know & $9(18.4)$ & $40(81.6)$ & \\
\hline \multicolumn{4}{|c|}{ Do you have sufficient knowledge about MERS-CoV? } \\
\hline Yes & $168(53.3)$ & $147(46.7)$ & \multirow[t]{3}{*}{0.082} \\
\hline No & $85(38.6)$ & $135(61.4)$ & \\
\hline Don’t know & $2(40)$ & $3(60)$ & \\
\hline \multicolumn{4}{|c|}{ Do you have contact with MERS patient? } \\
\hline Yes & $38(73.1)$ & $14(47.9)$ & \multirow[t]{3}{*}{0.428} \\
\hline No & $217(46.5)$ & $250(53.5)$ & \\
\hline Don’t know & 0 & $21(100)$ & \\
\hline
\end{tabular}

Table 4: Attitude of Health Colleges' students toward MERS-CoV

\begin{tabular}{lc}
\hline Item & Participants' response N. (\%) \\
\hline Are you worried about one of your family members can get infection? & $424(78.5)$ \\
Yes & $126(21.5)$ \\
No & $278(51.5)$ \\
Transmission of MERS can be prevented by using standard and isolation precautions given by CDC, WHO, APIC, etc? \\
Yes & $272(48.5)$ \\
No & $252(46.7)$ \\
Prevalence of MERS can be reduced by active participation of health care worker in hospital infection control program? \\
Yes & $298(53.3)$ \\
No & \\
If MERS-CoV vaccine is available, would you take it? & $332(61.5)$ \\
Yes & $218(38.5)$ \\
No & \\
Intensive treatment should be given to diagnosed patients? & $390(72.2)$ \\
Yes & $150(27.8)$ \\
No & $415(76.9)$ \\
Healthcare workers must acknowledge themselves with all the information about the virus? & $135(23.1)$ \\
Yes & \\
No & $452(83.7)$ \\
Is the available information about MERS-CoV in Saudi Society sufficient? & $98(11.6)$ \\
Yes & \\
No & $498(92.2)$ \\
Does the government institutions able to control the epidemic? & $42(7.8)$ \\
Yes & \\
No &
\end{tabular}


Regarding attitude, the results showed that $>50 \%$ of students exhibited a positive attitude toward MERS-CoV (Table 4). Almost $84 \%$ and $92 \%$ of students felt that the information in Saudi society was sufficient to deal with MERS-CoV and that the government could be able to control any emerging MERS epidemic, respectively. However, only $47 \%$ and $52 \%$ of students realized that MERS infection could be prevented by active participation of health care worker in hospital infection control program and by using isolation and standard precautions, respectively.

\section{Discussion}

The role of students in Health Colleges as health advocates necessitates them to be more informed and prepared to raise awareness in their communities (AlMohrej and Agha, 2017). Moreover, the knowledge and attitude among these students might have positive effects on their future healthcare practice when dealing with MERS-CoV infections (Stirling and Harmston, 2015). Thus, this study was conducted targeting Health colleges' students, as potential health workers of the future, with the aim of investigating their knowledge and attitude towards MERS-CoV.

With regard to the student's source of information about MERS-CoV, the majority of students recorded that they depend on the social media $(57 \%)$, followed by $\mathrm{MOH}$ website $(32 \%)$ as a source of information about Coronavirus. These results are consistent with previous findings from Saudi Arabia (Al-Hazmi et al., 2018; Hassan, 2016; Salem et al., 2016). This is an expected finding owing to the widespread use of the internet in the last years. Most of the colleges' students become internet users and depend on it as the main source of information in many fields. This finding attracts the notice to the significance of internet utilization, especially social media in any health education campaign targeting Saudi students Hassan, 2016).

Although, 90\% of the participants knew that MERS$\mathrm{CoV}$ is a viral infection, yet nearly half of the participants have no idea about the incubation period $(49 \%)$ or the suitable line of treatment $(46 \%)$. Similar results were reported by Salem et al. (2016) who found that $63 \%$ and $51 \%$ of 672 medical students yielded incorrect answers when asked about MERS-CoV incubation period and treatment, respectively (AlTawfiq, 2013). This indicates the need to draw attention to such pieces of information in the curriculum of their microbiology course. Moreover, in awareness campaigns directed to medical students, there may be no enough coverage of these elements, which resulted in decreased awareness (Al-Mohrej and Agha, 2017).

It is recommended that the students educate themselves by reading the materials prepared by Centers for Disease Control and Prevention (CDC) and WHO, which cover the various aspects of the disease and shed light on the important elements of prevention and early recognition of the disease (CDC, 2015). However, only $52 \%$ of the participants were aware about the preventive measures as per international guidelines. Contrary to these findings, Al-Mohrej and Agha (2017) found that $96 \%$ of 136 medical students aware of the protective measures. The researchers thought this awareness was due to the teaching activities focusing on disease prevention and control in the hospital. In this context, a greater emphasis on infection control topics during the practical sessions is required for our students. Another possible explanation of such discrepant result is that all their study participants were medical students. In this study, only $22 \%$ of participants were medical students. It is noteworthy to mention that the highest percentage of sufficient knowledge in this study was obtained from medical students $(72.9 \%)$ versus $51.4 \%$ form medical laboratory and $46.2 \%$ from radiology students. This finding highlights the need to design educational courses on new emerging diseases, field epidemiology and public health to all Health Colleges' students to prepare them for dealing with these types of health emergencies in their future healthcare practice.

In this study, similar to previous reports, diarrhea comprised the least (52\%) known symptom (Al-Hazmi et al., 2018; Al-Tawfiq, 2013; Khairoalsindi et al., 2017). This might be attributed to that diarrhea is not stressed upon as a manifestation of MERS-CoV in awareness campaigns, as it is an atypical symptom for MERS-CoV (Al-Tawfiq, 2013).

There is a common public misconception for the availability of vaccination for MERS-CoV (Bawazir et al., 2018). In this study, $46 \%$ of students thought that there is available vaccine. Similar results were reported in previous studies (Al-Hazmi et al., 2018; Khairoalsindi et al., 2017). However, Al-Mohrej and Agha (2017) found that $92 \%$ of students knew that vaccines to MERS-CoV are not yet available and explained their finding due to the clinical studies and the awareness campaigns in their teaching hospital. This implies that educational courses to improve the students' knowledge of updated preventive measures should be added in the current curricula.

The present study showed that there is a significant difference among students of different ages in the knowledge score, with higher levels of knowledge among the older students. This finding is in support with previous studies which reported that the level of awareness increase with the years of experience (Alkot et al., 2016; Khairoalsindi et al., 2017; WHO, 2013). These findings must be taken into consideration during planning for any intervention program against Coronaviruses where people with low knowledge are more vulnerable to infection. Continuous awareness campaigns and additional educational activities for students, especially in the early years, regarding the contemporary issue of MERS-CoV is recommended. 
This study investigated the level of comprehension about the disease among health collages students. The findings suggest a good level of perception about the disease risk; $63 \%$ thought it could be fatal, $67 \%$ found that HCWs are at high risk of infection and $72 \%$ agreed that patients should receive intense course of treatment. Similar findings were observed by Al-Hazmi et al. (2018), who related it to the orientation of the students about one of the serious consequences of acquiring MERS-CoV infection. Students' attitude should be considered in designing any educational activities. A noteworthy finding is the strongly positive attitude of students to protect their families from catching infections and their willingness to take vaccine whenever available. The enthusiasm of the participants to get all information about the virus and their evident trust towards the efforts exerted by their governance are other important features of positive attitude. This will consequently affect the degree of response for any health education campaign targeting these students.

This study had some limitations. The questionnaire data may have been subject to recall bias and misclassification. However, we can expect that heath colleges' students have considerable medical knowledge to correctly respond to all items of the questionnaire tool. Besides, our study was limited to the southern Saudi region. Therefore, the results presented here may not be generalized to the rest of Saudi Arabia. Finally, the lack of detailed information about the knowledge and attitude of Health Care Workers (HCWs) towards MERS is a potential limitation of this study. Therefore, further large-scale studies from various Saudi regions are important to further explore the awareness and attitude of HCWs at the national level.

\section{Conclusion}

This study showed a good knowledge level and a strong positive attitude towards protection from MERS$\mathrm{CoV}$ among Health Colleges' students. However, there is a noticeable defect in the knowledge level about the microbiology of the virus and the infection control measures. Therefore, continuous awareness campaigns and additional educational activities for students, especially in the early years, regarding the contemporary issue of MERS-CoV, the microbiology of the virus, field epidemiology and updated preventive measures should be added in the current curricula.

\section{Acknowledgement}

The authors would thank The Deanship of Scientific Research and Health Colleges, Najran University for their help and support

\section{Author's Contributions}

Ahmed Morad Asaad: Was responsible for the conception and study design and has been involved in analyzing the data and writing as well as revising the manuscript.

Rehab H. El-Sokkary: Helped directly in constructing the study design along with writing and revising the manuscript.

Mahdi Ali Alzamanan: Helped directly in data collection, data analysis and writing the manuscript.

Abdullah I. Aedh and Fatma Omar Khalil: Helped directly in data analysis and writing as well as revising the manuscript.

\section{Ethics}

This article is original and contains unpublished material. Ethics approval was obtained from the Ethics and Research committee of Najran University. A written consent was obtained from all participants. The corresponding author confirms that all of the other authors have read and approved the manuscript and there are no ethical issues involved.

\section{References}

Aldrees, T., M. Al Ghobain, A. Alenezi, A. Saleh and A. Dana et al., 2017. Medical residents' attitudes and emotions related to Middle East respiratory syndrome in Saudi Arabia. Saudi Med. J., 38: 942-947.

Al-Hazmi, A., I. Gosadi, A. Somily, S. Alsubaie and A. Bin Saeed, 2018. Knowledge, attitude and practice of secondary schools and university students toward Middle East Respiratory Syndrome epidemic in Saudi Arabia: A cross-sectional study. Saudi J. Biol. Sci., 25: 572-577.

Alkot, M., A.M. Albouq, A.M. Shakuri and M.S. Subahi, 2016. Knowledge, attitude and practice toward MERS-CoV among primary health-care workers in Makkah Al-Mukarramah: An intervention study. Int. J. Med. Sci. Pub. Health, 5: 952.

Al-Mohrej, A. and S. Agha, 2017. Are Saudi medical students aware of middle east respiratory syndrome coronavirus during an outbreak? J. Infect. Pub. Health, 10: 388-395.

Al-Mohrej, O.A., S.D. Al-Shirian, S.K. Al-Otaibi, H.M. Tamim and E.M. Masuadi et al., 2016. Is the Saudi public aware of Middle East respiratory syndrome? J. Infect. Pub. Health, 9: 259-266.

Al-Tawfiq, J.A., 2013. Middle East respiratory syndrome-coronavirus infection: An overview. J. Infect. Pub. Health, 6: 319-322.

Bawazir, A., E. Al-Mazroo, H. Jradi, A. Ahmed and M. Badri, 2018. MERS-CoV infection: Mind the public knowledge gap. J. Infect. Pub. Health, 11: 89-93. 
CDC, 2015. Updated Information and guidelines for evaluation of patients for Middle East Respiratory Syndrome Coronavirus (MERS-Co V) Infection. Centers for Disease Control and Prevention.

European Centre for Disease Prevention and Control, 2015. Rapid Risk Assessment. Middle East Respiratory Syndrome Coronavirus (MERS-Co V). 17th Update.

Hassan, H.M., 2016. Knowledge, attitude and belief of Al-Ghad College students toward Coronavirus Infection, Riyadh, Saudi Arabia. Int. J. Med. Res. Re., 4: 19-26.

Ibrahim, I.A. and Y.K. Alghamdi, 2016. Awareness, attitude and practices of students medical sciences, medicine and pharmacology Colleges towards MERS-CoV Infection: A Cross Sectional Study. Sav. J. Med. Med. Sci., 2: 127-141.

Khairoalsindi, O.A., A.M. Tayeb, R.M. Mandora and M.I. Siddiqui, 2017. Middle East respiratory syndrome coronavirus: Are Makkah medical students sufficiently aware of it? Int. J. Adv. Res., 5: 1743-1751.

Khan, K., J. Sears, V.W. Hu, J.S. Brownstein and S. Hay et al., 2013. Potential for the international spread of Middle East respiratory syndrome in association with mass gatherings in Saudi Arabia. PLoS Curr.

Lwanga, S.K. and S. Lemeshow, 1990. Sample size determination in health studies. Geneva: World Health Organization.
Memish, Z.A., A. Zumla, R.F. Alhakeem, A. Assiri and A. Turkestani et al., 2014. Hajj: Infectious disease surveillance and control. Lancet, 383: 2073-2082.

Salem, I., J. AL Ahmada, B. Abalkhail, S. Howladar and H. Balto et al., 2016. Knowledge and attitude about Middle East respiratory syndrome Coronavirus (MERS-CoV) among medical students in king Abdulaziz University in Jeddah, 2015. Int. J. Curr. Res., 8: 28733-28740.

Saudi Ministry of Health, 2018. FAQs Coronavirus (MERS-CoV).

Stirling, B.V. and J. Harmston, 2015. Readying nurses for clinical practice: Protecting students during an outbreak of Middle Eastern-Coronavirus in Saudi Arabia. J. Nurs. Edu. Practice, 5: 40-44.

STROBE, 2007. STROBE checklist, Version 4, published in Oct/Nov 2007.

WHO, 2013. EMRO. Middle East Respiratory Syndrome Coronavirus: Joint Kingdom of Saudi Arabia. Riyadh: World Health Organization Mission, pp: 4-9.

WHO, 2018. Frequently asked questions on Middle East respiratory syndrome coronavirus (MERS-CoV), World Health Organization.

Zaki, A.M., S. Van Boheemen, T.M. Bestebroer, A.D. Osterhaus and R.A. Foucheir, 2012. Isolation of a novel coronavirus from a man with pneumonia in Saudi Arabia. N. Engl. J. Med., 367: 1814-1820. 